\title{
ACTUALIZACIONES
}

\section{Políticas de salud o políticas insalubres? De la higiene a la salud pública en Colombia en la primera mitad del siglo $X X$}

\author{
Emilio Quevedo
}

Este trabajo incluye los resultados iniciales de un proyecto de investigación mucho más amplio que vengo desarrollando como investigador del Instituto de Salud en el Trópico de la Universidad Nacional de Colombia. Dicho proyecto se centra en el estudio de la lucha entre las grandes metrópolis europeas y norteamericanas (Francia, Inglaterra y Estados Unidos) por la hegemonía en el campo de la salud pública y la medicina tropical en el Tercer Mundo durante las últimas tres décadas del siglo $\mathrm{XIX}$ y las primeras cinco del siglo $\mathrm{XX}$, así como del papel de los actores e instituciones locales receptoras a nivel local. El proyecto pretende explorar las características de dicho proceso en Latinoamérica (México, Colombia y Brasil) y compararlas con las ocurridas en India y Australia, con el fin de reconstruir la dinámica de las relaciones científico-técnicas entre las metrópolis y los espacios geoculturales periféricos.

El trabajo concreto que se presenta corresponde a un enfoque preliminar del caso colombiano y se centra exclusivamente sobre el análisis de cómo las características del contexto internacional influyeron en el campo de la salud en nuestro país durante las últimas dos décadas del siglo XIX y las primeras cinco del X.

La recepción y asimilación local de la salud pública metropolitana, así como su proceso de institucionalización, las interacciones con los actores locales, las reacciones y conflictos sociales y políticos que ocurrieron en consecuencia, no se analizan aquí. Un enfoque histórico y sociológico de este aspecto del problema, así como el análisis comparativo con las particularidades de dicho proceso en las otras regiones de América Latina, Asia y Australasia que se han incluido en el proyecto global, serán discutidas en futuros artículos y en un libro que contendrá mucho más material comparativo adicional y una elaboración más detallada de cada uno de los aspectos aquí tratados, a partir del análisis de fuentes documentales más amplias.

\section{ANTECEDENTES}

\section{La salud pública en Colombia al terminar el siglo $\mathrm{XIX}$}

El concepto de higiene, que durante el siglo XIX fundamentaba las actividades de salud pública en Colombia $(1,2)$, clasificaba las enfermedades en agudas, las cuales se entendían como causadas por el encuentro fortuito entre el individuo y los miasmas, sustancias pútridas suspendidas en el aire que surgían de las aguas estancadas y malolientes de los pantanos, (3, 4) y crónicas, las cuales se entendían como el resultado de los hábitos personales y las formas de vida (5). Así, la higiene pública, considerada como responsabilidad del Estado, estaba encargada del problema público de la prevención de las enfermedades agudas y sus causas. La

Subdirector de Investigación y Desarrollo, Instituto Nacional de Salud; Profesor asociado, Instituto de Salud en el Trópico, Facultad de Medicina, Universidad Nacional de Colombia, Santa Fe de Bogotá. 
higiene privada, encargada de la prevención de las crónicas, era en cambio un asunto de la vida privada de los individuos.

El estado colombiano, entonces, tomaba bajo su responsabilidad aquellos problemas catalogados desde tiempo atrás bajo el concepto de higiene pública (6). En el campo de la higiene privada, el Estado sólo debía preocuparse por las acciones de instrucción pública con el fin de inculcar en el pueblo los preceptos de la higiene personal y la urbanidad (1). La recuperación de la salud en caso de enfermedad y, por tanto, el tratamiento de las enfermedades se concebía como un problema de los individuos mismos, en tanto que hacía parte del ejercicio de su propia libertad. Dicho problema no tenía ninguna relación con la esfera del Estado. Este, sólo tenía que garantizar que el nivel técnico y jurídico de la práctica médica fuese el adecuado (7).

A pesar de esos planteamientos, durante todo el siglo no existió en nuestro país una estructura sanitaria estatal realmente capaz de asumir en la práctica el cumplimiento de tales propuestas, y el problema no parece ser simplemente ausencia de una capacidad técnica, la cual es innegable, sino también las dificultades del Estado y de la dinámica socioeconómica del país en su totalidad.

No obstante, durante los años comprendidos entre el final de la Guerra de los Mil Días y la Primera Guerra Mundial, se producen cambios destacados en nuestra economía. Así, la consolidación de la economía cafetera, el desarrollo industrial urbano y la apertura de vías férreas (8) crearon nuevas formas de asentamiento y de movilización demográfica que potenciaron nuevas patologías tropicales, no comunes en los años anteriores, o aumentaron las ya existentes y conllevaron necesariamente un cambio de actitud en el Estado colombiano frente a la salud de los pobladores y su traducción en decisiones políticas.

Pero, el cambio no se debió sólo a una modificación de las condiciones sanitarias internas ni a la mutación en una actitud nacional sino, y de manera muy importante, a la confluencia de una serie de eventos y circunstancias internacionales que se venían configurando desde finales del siglo XIX y comienzos del $X X$ y que favorecieron la intervención extranjera en el proceso de estructuración de la organización sanitaria nacional.

Tales eventos y circunstancias fueron: 1) el desarrollo del comercio internacional y el papel protagónico cada vez más destacado de los Estados Unidos en esta carrera en América Latina; 2) la competencia mundial de este país por el monopolio y la explotación del petróleo en los países periféricos como fuente de riqueza y de energía; 3 ) la creación de las fundaciones filantrópicas norteamericanas, más específicamente, la Fundación Rockefeller; 4) la constitución y consolidación de las organizaciones sanitarias internacionales, especialmente, la de la Oficina Sanitaria Internacional; 5) el desarrollo en Norteamérica de una nueva manera de entender el concepto de salud pública y, como inmediata consecuencia, 6) la constitución e institucionalización de una nueva especialidad científica, poseedora de una nueva concepción de su práctica. Aunque algunos de estos eventos ya han sido estudiados en general y de forma aislada y puntual, pretendemos hacer un enfoque integral de su interacción en el país. Analizaremos cada una de ellas y sus influencias en el campo de la salud en Colombia.

\section{LA EXPANSION ECONOMICA NORTEAMERICANA Y LA TRANSICION DE LA HIGIENE A LA SALUD PÚBLICA}

\section{Petróleo y expansión comercial norte- americana}

Los años que siguieron a la Guerra Civil norteamericana, 1865-1914, fueron testigos del incremento absoluto que tuvo lugar en la producción estadounidense hasta 1929, que superó con creces el de las mayores potencias industriales del mundo, convirtiéndose en la primera potencia industrial del planeta (9) y que requirió de la explotación de los recursos 
naturales y materias primas propias y de otros continentes, así como de la apertura de amplios mercados internacionales.

Así, desde la primera década del siglo XX (10), la avalancha de empresarios norteamericanos que codiciaban nuestros recursos no se hizo esperar y la explotación petrolera será sin duda una de las actividades más destacadas. En 1920, Harding, presidente de los Estados Unidos, proclamaba que "llegará el día en que la hegemonía mundial pertenezca a la nación que posea petróleo y sus derivados" (11). Igualmente, en 1919, el Departamento de Estado de Estados Unidos había declarado: "la importancia vital de asegurar abastecimientos suficientes de petróleo para las necesidades actuales y futuras de los Estados Unidos es un punto que ha merecido la especial atención de este departamento. Se recomienda prestar toda ayuda legítima a los ciudadanos o a los intereses de los Estados Unidos que buscan concesiones o derechos sobre petróleo" (11). De la misma manera, en 1929, la Junta Federal de Reservas Petrolíferas de los Estados Unidos consideraba conveniente que los Estados Unidos adquirieran yacimientos petrolíferos en diversas partes del mundo, especialmente en las naciones de América Latina (11).

En este contexto, John D. Rockefeller (18391937) logró crear el mayor trust petrolero del mundo, la Standard Oil Company (12), dominando no sólo la comercialización del petróleo sino también las compañías ferrocarrileras y trasportadoras terrestres relacionadas con la distribución del crudo, ejerciendo su dictadura sobre el $95 \%$ del mercado del petróleo mundial $(12,13)$.

Pero, para subsanar el impase que representaba el monto proporcional de impuestos, Rockefeller creó pequeñas empresas de explotación de productos renovables y no renovables en Latinoamérica, Africa y Asia, en donde encontraría economías débiles, generalmente agrícolas y con necesidades sentidas para el proceso de industrialización. Tales empresas asegurarían la disminución de impuestos e, incluso, la exención de los mismos en los países sede. La Standard Oil Company instala así múltiples empresas pequeñas a través de contratos con el Estado o con particulares con capacidad de inversión.

En Colombia, las concesiones petroleras que habían sido entregadas en 1905, por el presidente, general Rafael Reyes, al general Virgilio Barco, la primera, y a Roberto de Mares, la segunda, fueron vendidas a compañías norteamericanas en 1916, una vez terminada la Primera Guerra Mundial. La de Mares fue negociada con tres testaferros de la Standard Oil (Bendum, Trees y Crawford), quienes fundaron la Tropical Oil Company $(11,12,14)$. Igualmente, la Concesión Barco pasó, en 1918, a manos de la Gulf Oil Company, y se convirtió en la Colombian Petroleum Company (11). De otro lado, la United Fruit Company, creada en 1899 con capital americano y cuyos cuarteles generales se ubicaban en Boston, había establecido sus enclaves bananeros en Centroamérica y en la costa atlántica colombiana, en la llamada zona bananera del departamento de Magdalena (15-17).

Esta expansión internacional traía consigo el problema de asegurar la salud de los trabajadores norteamericanos en estas inhóspitas e insalubres regiones tropicales y el tema de la salud se fue convirtiendo en una preocupación importante de los empresarios y del Estado nortamericano. Era necesario, por una parte, impulsar el control sanitario en los puertos a donde llegasen barcos comerciales y militares norteamericanos y desde donde saliesen naves comerciales extranjeras hacia los Estados Unidos y, por otra parte, sanear las zonas continentales en donde se venían estableciendo dichos enclaves petroleros y fruteros. Surgieron, así, dos grandes estrategias: en primer lugar, la implementación de una intervención estatal tendiente a la creación de una oficina sanitaria de carácter internacional encargada de coordinar los programas sanitarios adecuados en los países periféricos $y$, segundo, el apoyo por parte del capital privado a la investigación médica sobre enfermedades tropicales y a la intervención sanitaria directa en dichos países. 


\section{El papel del Estado norteamericano en el desarrollo de las nuevas organizaciones sanitarias}

Los intentos de una nueva organización sanitaria se habían venido dando desde la Primera Conferencia Sanitaria Internacional reunida en París en 1851, pero, debido a las múltiples dificultades de aunar criterios y conocimientos técnicos, solamente hasta 1912, la Decimosegunda Conferencia Sanitaria Internacional suscribirá una convención que reglamente la cuarentena para el cólera y la peste. Sin embargo, en ella escasamente se mencionarán la fiebre amarilla y la malaria, principales problemas de salud que entorpecen el desarrrollo de los enclaves económicos norteamericanos en América Latina (18).

Ya en 1880, el congreso estadinense había autorizado al presidente de los Estados Unidos para convocar la Quinta Conferencia Sanitaria Internacional en la ciudad de Washington "con la finalidad de organizar un sistema internacional de notificación en cuanto a la verdadera situación sanitaria de los puertos y plazas" con los cuales esta nación ejerce su comercio (19). Nuevamente, la divergencia de intereses y de realidades en el campo de las prioridades de salud pública entre Estados Unidos y Europa no permitieron llegar a acuerdos sustanciales durante esta reunión.

Sin embargo, el gobierno norteamericano continuó con su política y convocó a la Segunda Conferencia Sanitaria Internacional de las repúblicas americanas en Ciudad de México, para 1901, en cuyas conclusiones se recomendó que se convocase a una convención de representantes de las administraciones sanitarias americanas para formular acuerdos y reglamentos sanitarios con el objeto de poder reducir al mínimo los requisitos de cuarentena respecto del cólera, la fiebre amarilla, la peste bubónica, la viruela y otros brotes pestilíferos de gravedad (19). El método de la cuarentena que había sido impuesto por las organizaciones sanitarias europeas se había constituido en importante obstáculo para el comercio internacional, pues, obligaba a los barcos a permanecer por varios días anclados en los puertos antes de poder desembarcar con el fin de asegurar que no eran portadores de enfermedades infecciosas peligrosas para el bienestar de las poblaciones.

Esta nueva convención se celebró efectivamente en México en 1902 y el comité organizador se convirtió en la Oficina Sanitaria Internacional (la misma que más tarde, en 1947, se transformará en la Oficina Sanitaria Panamericana). Tres de sus siete miembros serían funcionarios norteamericanos, incluyendo al director general de sanidad de los Estados Unidos. Esto aseguraría el control norteamericano la nueva organización sanitaria (20).

Un ejemplo claro de esta hegemonía norteamericana durante dicho período lo constituyó el doctor Hugh S. Cumming, quien desempeñaba los siguientes cargos para esta época: director del Servicio de Salud Pública de los Estados Unidos (1920-1936), director de la Oficina Sanitaria Panamericana (1920-1947), miembro directivo de la Liga de Sociedades Nacionales de la Cruz Roja (1919-1923), miembro directivo del OIHP (1924-1946), miembro del Comité de Higiene de la Liga de las Naciones (1924). En pocas palabras, participó en todas las organizaciones sanitarias inter-nacionales del momento y manejó la salud pública norteamericana durante 16 años.

Más adelante veremos como esta nueva organización sanitaria actúa en Colombia, pero, miremos primero la segunda estrategia.

\section{Petróleo, filantropía, investigación médica y salud pública}

Simultáneamente a todos estos movimientos de carácter estatal, Rockefeller andaba muy preocupado, pues, los métodos competitivos que había utilizado para desplazar del mercado a sus competidores y asegurar la consolidación de la Standard Oil, le habían hecho acreedor a la mala fama de "barón ladrón". Sin embargo, en su condición de estudioso del evangelio y como fiel miembro de la iglesia bautista que era, conocía muy bien el versículo / de San Pedro, que decía: "La caridad puede ocultar muchos pecados" y con el paso de los años le iba aumentando el sentimiento místico de que había 
sido elegido personalmente para ser el frágil depositario de aquella gran fortuna para el bien de la humanidad. Por tanto, debía ganar más y más dinero para emplearlo en bien de sus semejantes (13).

Esta actitud se correspondía con dos de los principios de la más pura tradición luteranabautista: en primer lugar, el reconocimiento de que el único modo de vida grato a Dios es "no la superación de la moralidad terrena por medio de la ascesis monástica, sino precisamente el cumplimiento en el mundo de los deberes que a cada cual impone la posición que ocupa en la vida y que, por lo mismo, se convierte para él en profesión" (21) y, en segundo lugar, que "si Dios os muestra un camino que os va a proporcionar más riqueza que siguiendo camino distinto (sin perjuicio de vuestra alma ni de la de los otros) y lo rechazáis para seguir el que os enriquecerá menos...os negáis a ser administradores de Dios y a aceptar sus dones para utilizarlos en su servicio cuando El os lo exigiese" (21).

Estas convicciones religiosas y éticas, condujeron a Rockefeller hacia la filantropía. Esta actitud no era nueva en los Estados Unidos. La ausencia de una política estatal fuerte con relación a la educación, notoria después de la Guerra Civil norteamericana, hizo que las grandes instituciones universitarias de ese país se organizaran bajo la éjida del capital privado. Puede decirse que en lo referente al patrocinio de la educación y de la organización de una producción de expertos en distintos campos del saber, las fundaciones filantrópicas habían jugado en Estados Unidos el rol que el Estado había desempeñado en Europa (22). Pero, la filantropía no sólo venía a reforzar las ideas de participación democrática de la comunidad en la construcción de la nación norteamericana planteadas por su constitución sino que era también un buen negocio para el capital industrial norteamericano. Además, los donativos hacían bajar los impuestos, al apoyar el desarrollo de una universidad fuerte y de alto nivel académico se estaba asegurando la formación de un personal calificado que pudiese trabajar en la cada vez más compleja industria mecanizada.
Rockefeller inició su gran carrera filantrópica contratando al reverendo bautista Frederick T. Gates, quien fue introduciendo poco a poco el principio de la filantropía científica, descartando rápidamente -casi del todo- la caridad al detalle para dedicarse con toda confianza y regocijo al terreno de la filantropía al por mayor (13).

Gates comprendió rápidamente que la organización filantrópica era, además, una buena alternativa económica, más aún si se manejaba con criterio comercial. Por una parte, los donativos filantrópicos resultaban más baratos que los impuestos que había que pagar al Estado; pero, por otra, dichos donativos producian gran rentabilidad (13). De igual manera, la organización filantrópica era una buena manera de hacer inversiones en el terreno de la salud tanto al interior del país como en aquellos enclaves extranjeros tan caros a la Standard Oil y las compañías fruteras.

Así, Gates empezó por inducir la creación del Instituto Rockefeller para la Investigación Médica, en 1901, que continuaría las pautas del Instituto Pasteur de París y del Instituto Koch de Berlín. Luego, en 1903, Rockefeller creará la Comision General de Enseñanza, la cual, a partir de 1906, aunará sus intereses y esfuerzos a los del instituto. Una de sus principales realizaciones será la puesta en marcha de la reforma de la educación médica estadinense, propuesta por Abraham Flexner en su informe de 1910, tomando como insitución modelo a la Universidad de Johns Hopkins, de acuerdo con las recomendaciones del propio Flexner (13).

Luego, en 1909, crea la Comisión Sanitaria Rockefeller y el magnate instará a sus miembros a que escojan como blanco de trabajo una enfermedad, que afecte a gran número de personas, de la cual se pueda decir que se conocen todos los detalles y que pueda ser curada, no en el cincuenta u ochenta por ciento de los casos, sino en todos "...de tal modo que la gente -el hombre ordinario- pudiera ver los buenos resultados de la salubridad pública" (23). El doctor Charles Stiles, que venía investigando sobre el papel del anquilostoma (uncinaria) como causante de la anemia que 
producía un grave letargo en los trabajadores de las plantaciones sureñas de algodón, recomendó tomar la uncinariasis como modelo de la primera campaña. Efectivamente, era una enfermedad que afectaba a millones de personas y podía fácilmente curarse y prevenirse con cincuenta centavos por persona (13).

Pero Gates, que aspiraba a una institución que tuviese mayor impacto y que fuese la obra filantrópica por excelencia, perfiló en 1910 el proyecto de la creación de la Fundación Rockefeller, institución que asumiría la responsabilidad mundial del manejo de la fortuna Rockefeller destinada a la filantropía $(13,24)$. Será, entonces, el hijo del magnate, John D. Rokefeller, Jr., quien con Gates, se ponga a la cabeza de las actividades de la fundación, retomando la lucha contra las enfermedades que asolaban los países tropicales y los hacían inhóspitos para las influencias misionales y los enclaves empresariales portadores del modelo norteamericano de civilización. Se continuó, en primer lugar, la campaña contra la uncinariasis, para luego derivar los esfuerzos hacia la erradicación de la fiebre amarilla y más tarde de la malaria. La primera campaña contra la uncinariasis la realizó la Comisión Sanitaria Internacional en la región de Texas, al sur de los Estados Unidos donde el problema era de alta incidencia, y arrojó los mejores resultados. Por eso, como punto de partida, la fundación comenzó a difundir su acción por todos los continentes con dicha campaña (23).

Estos resultados hicieron que Rockefeller se interesase en el desarrollo de la salud pública como nueva disciplina médica. En los comienzos del siglo $X X$, la práctica de la higiene pública norteamericana se ubicaba en el marco de referencia de las concepciones higienistas europeas. Pero, la aparición de la medicina de laboratorio en la Europa de finales del siglo XIX, con sus grandes avances en la determinación de los agentes causaies de las enfermedades, fueran estos microbiológicos, químicos o físicos, define el nacimiento de una nueva epidemiología que desplazará a la antigua idea de higiene e impulsará el desarrollo de nuevas medidas terapéuticas masivas y efectivas para la erradicación de enfermedades. En este nuevo marco conceptual, de carácter unicausal, se piensa que una vez conocido el agente etiológico de una enfermedad, la higiene se encargará de determinar cuáles son los factores que facilitan o dificultan la acción del mismo y, por tanto, la aparición de la enfermedad y, a partir de ese momento, se pueden definir medidas para erradicarlo y, así, controlar el padecimiento.

Esto era precisamente lo que se proponía realizar la Fundación Rockefeller con la Campaña de Uncinariasis: demostrar la efectividad de la higiene, al actuar sobre los factores que favorecen la aparición de las enfermedades a un costo relativamente bajo frente a las pérdidas económicas que tales enfermedades ocasionan y, así, sensibilizar positivamente a la opinión pública hacia una reforma sanitaria y hacia las demás campañas sanitarias que iría poniendo en marcha. Si esto se lograba, las poblaciones de los países periféricos aceptarían facilmente las actividades económicas de la Standard Oil Company en la medida en que venían acompañadas por las acciones sanitarias de la Fundación Rockefeller.

Esta primera campaña, aunque exitosa por los resultados locales, no logró cabalmente estos dos objetivos y la uncinaria fue perdiendo su posición prioritaria en las decisiones de la fundación. Por eso, desde 1916, el esfuerzo se va derivando hacia la lucha contra la fiebre amarilla (25). El descubrimiento de oro en California, en 1848, había precipitado la importancia estratégica del istmo de Panamá como lugar de paso de americanos y europeos hacia el oeste de los Estados Unidos. En 1862, comenzó a pensarse en la posibilidad de un canal que cruzase el istmo. Después de la Guerra de los Mil Días y como consecuencia de un conflicto bastante complejo, en 1903, se produce la toma de Panamá por parte de los Estados Unidos y su separación de Colombia, convirtiéndose el istmo en zona estratégica norteamericana desde el punto de vista político, comercial y militar (26).

La apertura del canal, en 1914, aumentó la posibilidad de la exportación de la fiebre amarilla desde el Caribe hacia los Estados Unidos y el 
Asia tropical. En los primeros años del nuevo siglo, Carlos Finlay había descubierto el papel del mosquito Aedes aegypti, habitante urbano, en la transmisión de esta enfermedad y los métodos de control que él había puesto en marcha en La Habana y sus buenos resultados, hacían pensar que era posible erradicar la enfermedad, eliminando los mosqusitos que la transmitían. Se iniciaron campañas en Brasil, Ecuador, América Central, México y Colombia (25). Pero, el descubrimiento por parte del colombiano Roberto Franco, en 1907, de que la enfermedad podía ser transmitida también por otros mosquitos selváticos difíciles de eliminar (27) y su confirmación y aceptación por parte del doctor Soper en 1935 (25, 27), hicieron pensar a las directivas de la fundación que sus dos objetivos iniciales tampoco se lograrían en este campo y derivaron sus esfuerzos hacia la lucha antimalárica (25), con logros y descalabros similares.

Pero, así las cosas, las campañas de erradicación tenían que ser complementadas con otras acciones y la acción de la Fundación Rockefeller no podía terminar allí. Los Estados Unidos se habían quedado rezagados con respecto a Europa en cuanto a programas especiales de educación para las actividades de salud pública (28) y un nuevo tipo de especialidad médica, preparada para desarrollar este nuevo tipo de actividades sanitarias, se hacía evidentemente necesaria. Rockefeller tenía ya claro que era la fundación la institución llamada a impulsar el proceso de constitución e institucionalización de dicha disciplina.

Por eso, el 16 de octubre de 1914 se llevó a cabo una reunión en las oficinas de la Comisión General de Educación sobre capacitación para el servicio de salud pública. A esta reunión asistieron, entre otros, Simón Flexner (director del Instituto Rockefeller para la Investigación Médica), Wickliffe Rose (director del Consejo de Salud Internacional de la Fundación Rockefeller), Milton Rosenau (destacado salubrista norteamericano), William Welch (decano de la Escuela de Medicina de la Universidad de Johns Hopkins) y Abraham Flexner (ideólogo de la reforma de la educación médica en los E.U.A).
Rose y Welch, comisionados para formular un plan para crear un instituto de higiene que permitiera poner a los Estados Unidos al día con los otros países del mundo en ese campo (29), rindieron su informe en 1916 proponiendo un nuevo concepto de salud pública que integraba las versiones inglesa y alemana del concepto. Los ingleses tenían una mayor experiencia en la administración de servicios de salud y los alemanes una mayor trayectoria en la investigación de laboratorio en el campo de las enfermedades infectocontagiosas. En el nuevo instituto, deberían formarse salubristas integrales que estuvieran en capacidad de manejar ambos campos, para que pudiesen participar activamente tanto en el conocimiento y el control de las enfermedades como en la organización y manejo de los servicios de salud (29). Paso seguido, la Fundación Rockefeller apoyará la creación de una escuela de salud pública en la Universidad de Johns Hopkins, crisol de la reforma de la educación médica norteamericana ya impulsada por la misma Fundación Rockefeller. Luego, se crearon otras escuelas similares en Yale, Columbia y Harvard. Buena parte de los salubristas colombianos y latinoamericanos que participaron en los procesos de modernización de la salud pública en nuestros países, se formaron allí con apoyo de la fundación.

Así, el antiguo modelo higienista predominante en América Latina desde los tiempos de la conquista española, sería progresivamente dejado de lado y reemplazado por la nueva manera norteamericana de entender el concepto de salud pública (2). Este nuevo concepto potenció el desarrollo del concepto de campaña sanitaria de erradicación, posibilitando una nueva forma de intervención sobre las condiciones de salubridad y sobre las enfermedades epidémicas infecciosas.

\section{LA INTERVENCION NORTEAMERICANA EN LA TRANSICION DE LA HIGIENE A LA SALUD PUBLICA EN COLOMBIA}

\section{Las Organizaciones Sanitarias Internacionales en Colombia}

A pesar de la condición feudal y agrícola de la Colombia del siglo XIX, desde la década de 
1820, el país ya clasificaba en segundo lugar, después de México, entre las naciones hispanoamericanas en las cuales existía un mercado para las exportaciones estadinenses y era, entre ellas, la principal exportadora hacia los Estados Unidos. Al entrar en la segunda década de este siglo, el país tiene ya frente a sí una serie nueva de exigencias en materia de salud, derivadas de la nueva situación productiva y de poblamiento que mencionamos atrás (30). Y, más aún cuando, a partir de 1925, los Estados Unidos, reconociendo su arbitrario proceder con relación a Panamá, entrega a Colombia U.S. $\$ 25^{\prime} 000.000$ como recompensa y le abre importantes créditos financieros, de tal forma que en los cinco años siguientes, llegan U.S. $\$ 2001000.000$ más, en calidad de empréstitos norteamericanos $(14,31)$. Estos dineros serán invertidos fundamentalemente en la apertura de vías de comunicación (carreteras, ferrocarriles y telégrafos), que implican el desplazamiento de grandes masas de trabajadores a regiones tropicales bajas, ardientes y malsanas, conllevando nuevos y serios problemas en el campo de la salud (32). Así, para 1929 , el $45,16 \%$ de la inversión extranjera estaba -obviamente- en el petróleo, el 20,16\% en los servicios públicos (incluidas las vías de comunicación) y sólo el 3,22\% en la industria (33).

Entre tanto, la presencia de los Estados Unidos en Colombia en el campo de la salud será cada vez más clara, así como lo está siendo en la economía y en la política. La Oficina Sanitaria Internacional (hoy Oficina Sanitaria Panamericana) había comenzado la creación de todo un espacio de organización independiente de Europa con incidencia directa sobre los países latinoamericanos, impulsando la constitución de organizaciones sanitarias nacionales sólidas, con capacidad de realizar actividades de sanidad portuaria y acciones territoriales de lucha contra las principales enfermedades epidémicas y endémicas y de imponer medidas sanitarias, acudiendo obviamente a los préstamos externos.

El Código Sanitario Panamericano, firmado en la VII Conferencia Sanitaria Panamericana, reunida en La Habana en noviembre de 1924, expresa claramente el propósito de estimular y proteger mejor la salud pública de sus respectivas naciones y, particularmente, a fin de que puedan aplicarse medidas cooperativas internacionales eficaces para impedir la propagación de las infecciones que son susceptibles de transmitirse a los seres humanos, y para facilitar el comercio y las comunicaciones marítimas internacionales (34).

Consecuentemente, Pablo García Medina, principal figura de la organización sanitaria colombiana, en el Informe Anual de la Junta Central de Higiene al Ministerio de Gobierno en 1917, confirmaba: "Si no emprendemos muy pronto estas obras llegará el día en que ninguno de los buques que hayan de pasar por el Canal de Panamá quieran tocar en nuestros puertos, para no verse sometido a la cuarentena de seis días que se mantendrá en Colón mientras las condiciones sanitarias de estos puertos no mejoren" (35). En el mismo sentido, insitía: "Cuando la guerra europea termine debemos estar preparados para las necesidades del comercio universal y para ello debemos hacer pronto toda clase de esfuerzos. Estamos colocados en este dilema: o quedamos excluidos del comercio, o algún poder extraño vendrá a imponernos las medidas sanitarias que la civilización exige" (35). Colombia firma, así, los acuerdos de salud interamericanos e inicia una carrera de transformación de su estructura sanitaria. Ya, en 1918, la Junta de Higiene se había convertido en Dirección Nacional de Higiene, la cual fue reorganizada en 1923 y, luego, en 1925, integrada al nuevo Ministerio de Instrucción y Salubridad Pública (1), para convertirse en el Departamento Nacional de Higiene y Asistencia Pública, ente autónomo y encomendado a la dirección de Pablo García Medina, en 1931. Finalmente, aparece en 1938 el Ministerio de Trabajo, Higiene y Previsión Social, del cual se desprenderá, en 1946, el Ministerio de Higiene (1).

Así, desde el punto de vista de la salud, se van configurando en ese momento dos Colombias: una que mira hacia adentro, manteniendo aún la idea de que la salud es un problema privado de los individuos, y formando médicos de 
orientación francesa anatomoclínica para que puedan atender a los cafeteros, a los incipientes industriales, a los funcionarios del Estado, a los campesinos y trabajadores del ferrocarril y del Ministerio de Obras Públicas, etc., mientras el Estado pone en marcha incipientes medidas sanitarias internas para evitar las enfermedades de los pobres.

La otra, volcada hacia el exterior, que comienza a interesarse por no quedarse por fuera de los procesos del mercado internacional, preocupándose por el saneamiento en los puertos y de las regiones continentales cercanas al mar, en donde se encuentran los asentamientos extranjeros (36).

\section{Las fundaciones filantrópicas norte- americanas en Colombia}

Además del papel jugado por las organizaciones sanitarias internacionales, la intervención extranjera se verá mas legitimada y garantizada con la participación de las emergentes fundaciones filantrópicas de los Estados Unidos. En este momento, el comercio y la explotación internacional de los recursos naturales de los países tropicales aparecen ya ante los actores locales como acciones civilizantes porque, ligados a ellas, vendrían las medidas sanitarias y los médicos salubristas norteamericanos especialistas en enfermedades tropicales que se encargarían del saneamiento de estas regiones. Todo esto coincide con los ya mencionados idénticos fines de la Standard Oil Company y de la Fundación Rockefeller. En este sentido, es clara la afirmación de Burton Hendrick, cuando refiriéndose a las primeras escuelas de salud pública y de enfermedades tropicales de los Estados Unidos, afirmaba, en 1915, que se crearon para preparar "a los exploradores americanos para lo que promete ser uno de los más grandes movimientos en la historia: la apertura completa de los trópicos a la civilización" (37).

El primer contacto de nuestro país con la Fundación Rockefeller se da en 1917 cuando, "por solicitud del Gobierno nacional", una comisión norteamericana financiada por el
Instituto Rockefeller, hace un estudio para determinar la presencia de fiebre amarilla en Colombia. El objetivo era el de definir la necesidad o no de la cuarentena impuesta a los barcos colombianos y a todos aquéllos que tocasen nuestras costas con rumbo al canal de Panamá, según las normas implantadas por la Oficina Sanitaria Panamericana (38).

La justificación de tal intervención fue expuesta por el doctor García Medina de la siguiente forma: "Los resultados que se obtuvieron con las campañas de sanidad que el Departamento de Higiene de los Estados Unidos emprendió en Cuba, Méjico y en Panamá, y los que consiguieron el doctor Oswaldo Cruz en el Brasil y el doctor Liceaga en Veracruz, indujeron a aquella benéfica institución (Instituto Rockefeller) a formar, con sus propios recursos, una comisión de eminentes higienistas encargada de visitar los lugares donde ha aparecido, en cualquier tiempo, fiebre amarilla, no solamente en la América del Sur, en las Antillas y la América Central, sino también en el Asia y en el Africa" (38).

Pero, de la composición de esta comisión, se traslucen claramente las intenciones y la conexión existente entre la organización filantrópica y el gobierno norteamericano. Sus miembros fueron: "Doctor William G. Corgas, mayor general del Servicio Sanitario de los Estados Unidos de América, director de los trabajos de sanificación en Cuba y Panamá, cirujano mayor del ejército norteamericano y presidente de la comisión; doctor Henry R. Carter, Inspector de Sanidad Marítima de los Estados Unidos y jefe del Servicio de Cuarentenas; doctor Teodoro C. Lyster, médico militar de los Estados Unidos y segundo jefe sanitario de la zona del canal de Panamá; doctor Eugene R. Whitmore, bacteriólogo del Servicio de Sanidad Americano, profesor de anatomía patológica y parasitología; doctor William Wrightson, ingeniero sanitario y secretario de la comisión; y doctor Juan Guiteras, jefe del Departamento de Sanidad de Cuba (38).

No hay dudas ni de las intenciones comerciales ni de la interacción entre el Estado norteamericano, las fuerzas militares y los intereses 
privados en la intimidad de la comisión. Tampoco del papel de los actores colombianos en favorecer la intervención, a pesar de la conciencia de la existencia de dichas intenciones.

\section{La campaña de uncinariasis en Colombia}

"De la misma manera que otros países lo habían solicitado", en 1919, el gobierno colombiano "solicita" a la Junta Internacional de Sanidad de la Fundación Rockefeller su cooperación para el establecimiento de la campaña de erradicación de la uncinariasis en nuestro país. En respuesta a tal petición, del 22 de diciembre de 1919 al 31 de enero de 1920, el doctor Luis Shapiro, médico representante de la Fundación Rockefeller, con el apoyo entusiasta y decidido del entonces Ministro de Agricultura y Comercio, doctor Jesús del Corral, de los médicos de la Academia de Medicina y en asocio de un facultativo y de 7 estudiantes de último año de medicina, llevó a cabo una investigación sobre la prevalencia de la anemia tropical o uncinariasis, la ascaridiasis y la trichuriasis, en el departamento de Cundinamarca. Este estudio dejó en claro que, de cada 100 personas, había 95 que albergaban alguno de los tres parásitos, entre un total de más de 8.500 personas (39).

Después del informe del doctor Shapiro, la Junta Sanitaria Internacional de la Fundación Rockefeller envió un comunicado al ministro, doctor Del Corral, aprobando la realización de la campaña en Colombia y precisando, entre otras cosas, las contraprestaciones que debería aportar el gobierno colombiano: por una parte, la organización de un Departamento de Uncinariasis, en el cual el representante de la junta desempeñará las funciones de director durante todo el período de cooperación, bajo cuyo arbitrio estarán todos los nombramientos, así como también la remoción de los empleados incompetentes y, por otra, la exención de derechos de aduana, el transporte de todo aquello que hubiese de emplearse en la campaña, el transporte de todos los empleados (con excepción del director) cuando estén en desempeño de sus funciones oficiales, franquicia postal y telegráfica para los asuntos oficiales e impresión de todo lo necesario y, finalmente, la adjudicación de una oficina central en Bogotá y oficinas en los distritos donde se establezcan trabajos (40).

Efectivamente, se crea el Departamento de Uncinariasis, por decreto número 261 de 1920 (36) el cual inicia sus actividades en la zona cafetera andina, en donde la uncinariasis era endémica. No se olvide que el café ocupa el primer rango -y casi el único- de las exportaciones colombianas a los Estados Unidos y que el café colombiano estaba catalogado en ese país como uno de los más suaves del mundo. El problema del saneamiento no sólo tuvo que ver entonces con los puertos y las zonas petroleras, sino también con las zonas de producción agrícola que eran de interés de los Estados Unidos.

Las actividades del Departamento de Uncinariasis se inician con programas tendientes al diagnóstico y tratamiento masivo contra la uncinariasis y al saneamiento, a través de la instalación de letrinas en las viviendas campesinas. La necesidad de modificar los hábitos higiénicos de los campesinos se hizo evidente, por lo cual se implementaron grandes campañas educativas y propagandísticas, con niños y adultos.

La campaña continuó hasta 1935 debido a tres acuerdos más entre el gobierno colombiano y la Fundación Rockefeller. Durante este período, el número de acciones aicanzado fue impresionante: para 1928 (noveno año de la campaña), George Bevier, director del Departamento o Sección de Uncinariasis durante 15 años, informa que la campaña "ha administrado un total de 2'530.853 tratamientos a 1'362.764 personas; ha distribuido 824.889 hojas impresas de distinta clase referentes a sus propósitos y dictado 231.564 conferencias y demostraciones públicas a las cuales han asistido, según cálculos cuidadosos, más de 4'000.000 de personas" (41). El total de letrinas instaladas y de actividades de saneamiento fue proporcional. Las cifras eran enormes, pero no se discutió, en ningún momento, la calidad de estas actividades. 
Pero, la colaboración de la Fundación Rockefeller y del departamento no se redujo exclusivamente al manejo de la uncinariasis: entre 1922 y 1923, la fundación contribuyó al estudio y control de la fiebre amarilla en Bucaramanga. En 1931 y 1932, aportó una suma para pagar el sueldo del director del Laboratorio Samper y Martínez. En 1932, sus técnicos comenzaron el estudio del paludismo. En 1933, participó en la creación del Laboratorio de Higiene Pública de Barranquilla y de la Escuela de Inspectores. En 1934, participó en la implementación de la Unidad Sanitaria de Pereira, la primera de su género en el país, y en estudios de fiebre amarilla rural y de la selva. En 1935, la campaña de uncinariasis se encaminó más al saneamiento del suelo y a partir de enero de 1936, aunque se entregó esta campaña completamente al cuidado del Departamento Nacional de Higiene, continuó la asesoría y financiación del laboratorio de Barranquilla y los trabajos de investigación en fiebre amarilla y paludismo, reunidos en la Sección de Estudios Especiales -uno de los antecedentes del Instituto Nacional de Salud- desde este mismo año (42).

Otra forma de incidir fue por medio de la formación de especialistas en salud pública en universidades norteamericanas asesoradas por la Fundación, los cuales ocuparán más tarde los principales cargos de la organización sanitaria nacional. Así mismo, la fundación facilitaba los medios para que los funcionarios del Departamento de Higiene visitaran los países donde mejor organizada estuviera la higiene, por medio de becas viajeras (42).

\section{El Servicio Cooperativo Interamericano de Salud Pública}

En los años 40, después de la Segunda Guerra mundial y durante la presidencia de F. D. Roosevelt, se inicia una nueva era de la influencia norteamericana en América Latina. Se establece un programa de cooperación interamericano entre el Instituto de Asuntos Interamericanos y las distintas naciones de América Latina. Este instituto ejercerá su participación en el campo de la salud a través del Servicio Cooperativo Interamericano de Salud Pública. En septiembre de 1942, el gobierno colombiano solicitará su participación y el señor Nelson Rockefeller visitará nuestro país, como representante de la Oficina de Relaciones Interamericanas. Un mes más tarde, el general doctor George Dunham, jefe de la División de Salud del Instituto de Asuntos Interamericanos, arriba a Colombia y celebra varias reuniones con los funcionarios de salud, con la asesoría del doctor John C. Bugher, jefe de la Sección de Estudios Especiales (llamada antes Departamento de Uncinariasis) y representante para Colombia de la Fundación Rockefeller. Con el doctor Dunham viene, además, el doctor Howard D. Shookhoff. El será el director del Servicio Cooperativo Interamericano de Salud Pública para Colombia, el cual deberá funcionar dentro del recién creado Ministerio de Trabajo, Higiene y Seguridad Social (43).

La nueva idea de salud pública había sido definitivamente institucionalizada en nuestro país y se separará definitivamente de la higiene con la creación del Ministerio de Salud Pública. Durante la década de los 50 , el Servicio Cooperativo Interamericano de Salud Pública afirmará su influencia dentro de este nuevo ministerio y se convertirá en su guía técnico, controlando el proceso de toma de deciciones en salud.

Por su parte, la actividad sanitarista de la Fundación Rockefeller había comenzado a declinar y en su agenda florecía el interés por apoyar las instituciones latinoamericanas de educación superior (25). Su mano derecha en Colombia sería, en principio, el Servicio Cooperativo Interamericano de Salud Pública el cual dedicará parte de sus actividades a incentivar la reforma de la educación médica en Colombia (44). Aunque esta reforma había sido recomendada anteriormente por la primera misión médica norteamericana (misión Humphrey), enviada por el Comité del Servicio Unitario en 1948 (45), la reforma definitiva comenzará después de la segunda misión médica norteamericana (misión Lapham), recomendada por la División de Salud del Instituto de Asuntos Interamericanos (46), la cual impulsará y organizará la implementación 
en Colombia del modelo de educación médica propuesto por Abraham Flexner, tal como se había hecho en los Estados Unidos desde 1910, con el apoyo de la fundación $(6,47)$.

La creación de la Facultad de Medicina de la Universidad del Valle será la consecuencia inmediata de esta segunda misión. Tendrá un modelo típicamente flexneriano mejorado con la inclusión de los departamentos de salud pública y medicina preventiva y será la plataforma para el impulso, tanto de esta universidad como de la reforma de la educación médica (1). El apoyo de la Fundación Rockefeller no se hace esperar, de la misma forma como lo haría en México con el Instituto Tecnológico de Monterrey y en Brasil con la Universidad de Sao Paulo, todas ellas instituciones ligadas a regiones de florecimiento industrial (25). Así, la Facultad de Medicina del Valle liderará el cambio definitivo de la educación medica colombiana en su tránsito al modelo flexneriano. Como punto de partida, en 1955, invitará a todas las otras facultades al Primer Seminario Nacional de Educación Médica, al cual asistieron, curiosamente, como observadores, representantes del gobierno, de la Oficina Sanitaria Panamericana, de la Fundación Rockefeller, del Servicio Cooperativo Interamericano de Salud Pública y de la Universidad de Tulane. Es decir, todas las entidades que tejían su red en torno a la reforma, orientada, como en los Estados Unidos, a preparar un médico acorde con las necesidades del desarrollo industrial y comercial nacional e internacional. En 1957, por invitación de la Facultad de Medicina de la Universidad de Antioquia, segundo baluarte de la reforma, se reunirá el Segundo Seminario Nacional de Educación Médica, en el cual el modelo de Flexner y el de la medicina preventiva y la salud pública, será ya el lenguaje común de todas las facultades de medicina del país. La institucionalización del modelo médico y salubrista norteamericano estaba en proceso de consolidación.

\section{CONCLUSIONES}

En este trabajo, hemos estudiado el tránsito desde la higiene hacia la salud pública en Colombia, en el marco de algunos eventos que ocurrieron a nivel internacional en el campo de la salud durante los últimos años del siglo XIX y la primera mitad del siglo $X X$, y que favorecieron el cambio gradual de Colombia, al igual que el de otras naciones latinoamericanas, desde la dependencia europea hacia la órbita de la "economía-mundo" norteamericana.

Tales eventos fueron, en primer lugar, la forma como el gobierno norteamericano, las organizaciones sanitarias internacionales y la Fundación Rockefeller, unieron sus intereses públicos y privados, políticos y comerciales, desarrollando un amplio espacio de organización sanitaria, paralelo al previamente establecido por Europa, con el fin de fortalecer su posicionamiento en Colombia. Este espacio se constituyó en marco de protección de las múltiples formas de comercio e inversión local, dentro del esquema de la economía de enclave.

En segundo lugar, la forma como el antiguo modelo higienista, predominante en Latinoamérica desde los tiempos del régimen colonial español y portugués, fue progresivamente dejado de lado y reemplazado por el concepto norteamericano de salud pública, desarrollado por las nuevas escuelas en este campo, creadas desde 1914 con el apoyo de la Fundación Rockefeller. Este nuevo concepto, construido con el fin de resolver necesidades sociales y políticas muy concretas, permite consolidar la idea de campaña erradicatoria, garantizando así una nueva posibilidad de intervención sobre las enfermedades infecciosas y epidémicas y se convierte en un instrumento potencialmente efectivo para mejorar la salud en los países periféricos, con el fin de crear condiciones adecuadas para el desarrollo de las actividades productivas y comerciales.

Como consecuencia, desde la creación del Departamento de Uncinariasis hasta la reforma de la educación médica, pasando por las campañas contra la fiebre amarilla y la malaria, la Fundación Rockefeller, las organizaciones sanitarias internacionales y el gobierno de los Estados Unidos, participan activamente en el proceso de toma de deciciones en salud en Colombia, determinando, en primer lugar, el tipo de organización sanitaria que el Estado deberá 
adoptar durante la primera mitad del siglo XX; en segundo lugar, la forma como se debe entender la salud pública como disciplina y sus formas de acción; y, tercero, el tipo de profesional de la salud que deberá formarse, en el marco de los nuevos modelos norteamericanos de salud pública y de educación médica.

Estos resultados nos obligan a no estar de acuerdo, en primer lugar, con aquéllos que creen que el problema del imperialismo cultural es solamente un problema de intervención económica y que lo cultural es consecuencia secundaria; y, en segundo lugar, con aquéllos que, como Lewis Pyenson, creen que el rol del imperialismo cultural se reduce sólo al fortalecimiento institucional, local que sirve de parapeto para la intervención política y que el papel del contexto sólo se reduce a la acción externa y circunstancial de los "siete pecados capitales" sobre las intenciones del científico y no sobre los saberes mismos y su forma de intervención sobre la realidad $(48,49)$.

Al contrario, nuestros resultados nos permiten afirmar, en primer lugar, que la intervención hegemónica de las metrópolis en las periferias no solamente implica formas de actividad económica y política, sino también acciones en el campo de la ciencia y de la cultura que no son simple consecuencia secundaria. Está claro aquí que la intervención cultural es instrumento fundamental para crear condiciones locales para la intervención económica y política posterior. En ese sentido, vale la pena recordar la afirmación de Marcos Cueto, que refuerza nuestros resultados, de que si bien, los programas de salud pública de la Rockefeller no siempre se llevaron a cabo exactamente en las áreas donde la Standard Oil Company tuvo sus asentamientos e inversiones, fue porque su proyecto hacía parte de todo un esfuerzo para asegurar la estabilidad del sistema capitalista internacional como un todo y para afirmar el rol político y cultural que los E.U.A. estaban comenzando a jugar en este sistema (25).

En segundo lugar, y confirmando las propuestas de Berger, Luckmann, Bloor, Latour y Woolgar, entre otros, que un concepto científico -en este caso, el de salud pública- no es solamente una representación mental rigurosa y sistemática de la realidad desde un punto de vista específico, sino también, y al mismo tiempo, una construcción social $(50,51)$ que incluye en su estructura íntima los intereses y tensiones contextuales $(52,53)$ y que es por eso, además, que puede convertirse en instrumento de hegemonía económica y cultural.

En tercer lugar, que el conocimiento no es una torre de marfil impenetrable desde el estudio social, por tener un estatuto especial y distinto del resto de formas del conocimiento como pensaron, Weber, Manheim, Marx y Merton (54).

En cuarto lugar, que el contexto no está compuesto sólo por las actitudes y tendencias malignas voluntaristas de las personas que sólo deben interesar al historiador de la ciencia cuando eso conduce a errores científicos, o por los roles sociales, la naturaleza del sistema de remuneraciones a los científicos, la competitividad, las demandas sociales por ciencia o por el sistema de normas según el cual se guían las acciones de los científicos, objeto de estudio del sociólogo funcionalista. El contexto consiste en todo el entretejido social y cultural de intereses y tensiones de la vida cotidiana que penetran hasta en lo más íntimo de la estructura de los conocimientos.

Finalmente, de manera frecuente se considera que la ciencia y la medicina han sido claros ejemplos de superioridad cultural de los países industrializados sobre el Tercer Mundo y se piensa que dicha superioridad garantizaría su carácter inherentemente progresivo. Por tanto, se supone que los países periféricos deberían sentirse afortunados y agradecidos porque dichos saberes y prácticas les sean transmitidos desde la metrópoli. Lo que en nuestro caso podemos concluir es que, si bien todas estas acciones metropolitanas favorecieron el tránsito de la higiene a la salud pública y apoyaron la modernización de los programas e instituciones sanitarias en Colombia, por ser ésta una nación que no tenía una política estatal explícita para los asuntos internacionales y en la que el manejo de éstos dependía del deseo y la voluntad de sus gobernantes (55), los resultados finales favorecieron más a los 
intereses extranjeros en la región que a la creación de una infraestructura institucional sólida que asegurase un cubrimiento y una solución clara de los propios problemas nacionales en salud.

Todo esto, además de que confirma las intuiciones de Teresa Meade y Mark Walker cuando proponen que las investigaciones sobre el rol de la ciencia y la medicina en una sociedad dominada pueden revelar importantes aspectos sobre el imperialismo cultural (56), nos abre un camino de investigación nuevo al unir distintas orientaciones de los estudios sociales y la historia de la ciencia para comprender de una manera más amplia e integral las dinámicas de la interacción científica entre las metrópolis y las periferias.

Pero, como bien dice Roy MacLeod, estos procesos de interacción son siempre de doble vía (57) y como remarca Chambers, no podemos olvidar que la ciencia siempre existe en asentamientos locales (58), el próximo paso hacia el futuro en nuestro trabajo, será estudiar los procesos de producción y organización de la salud pública en Colombia teniendo en cuenta el rol de los actores y de las fuerzas sociales locales, así como las condiciones contextuales específicas que interactuaron y negociaron con las tendencias del movimiento internacional que hemos descrito.

\section{Referencias}

1. Quevedo E, et al. La salud en Colombia: análisis sociohistórico. Bogotá: Estudio Sectorial de Salud, Ministerio de Salud, Departamento Nacional de Planeación; 1990.

2. Quevedo E, Hernández M, Miranda N. Ciencias médicas, estado y salud en Colombia: 1886-1957. En: Historia social de la ciencia en Colombia, volumen VIII. Bogotá: Colciencias; 1993:163-276.

3. Corbin A. El perfume o el miasma. El olfato y lo imaginario social, siglos XVIII y XIX. México; 1987.

4. Cipolla CM. Contra un enemigo mortal e invisible. Barcelona; 1993.

5. Laín P. El diagnóstico médico: historia y teoría. Barcelona: Salvat; 1982.

6. Quevedo E, Vergara A. El proceso de institucionalización de la educación médica en Colombia. Revista ECM (Bogotá) 1988;1(1):41-66.
7. Restrepo G, Villa A. Desarrollo de la salud pública colombiana. Medellín: Universidad de Antioquia, Escuela Nacional de Salud Pública; 1980.

8. Bejarano JA. La economía. En: Manual de Historia de Colombia, volumen III. Bogotá: Colcultura; 1980.

9. Degler CN. Historia de los Estados Unidos. EI desarrollo de una nación, 1860-1985, volumen II. Barcelona: Ariel; 1986.

10. Mesa D. La vida política después de Panamá. En: Manual de historia de Colombia, volumen III. Bogotá: Colcultura; 1980.

11. Villegas J. Petróleo, oligarquía e imperio. Bogotá: Tercer Mundo Editores; 1975.

12. Muñoz L. John D. Rockefeller, el filósofo del oro. Revista de Higiene (Bogotá) 1937;18(7):5-10.

13. Collier P, Horowitz D. Los Rockefeller. Barcelona: Tusquets Editores; 1986.

14. Pecault D. Orden y violencia: Colombia, 1930-1954, tomo I. Bogotá: Cerec; 1987.

15. LeGrand C. El conflicto de las bananeras. En: Tirado A, editor. Nueva historia de Colombia, volumen III. Bogotá: Editorial Planeta Colombiana; 1989:183-218.

16. Castrillón A. 120 días bajo el terror militar. Bogotá: Editorial Tupac-Amarú; 1974.

17. Gaitán JE. 1928: la masacre de las bananeras. Bogotá: Documentos y Testimonios; 1972.

18. Howard-Jones N. Problemas de la organización de la salud pública internacional entre las dos guerras mundiales. Primera parte. Crónica de la OMS 1977; 31:435-48

19. Howard-Jones N. La Organización Panamericana de la Salud: orígenes y evolución. Primera parte. Crónica de la OMS 1980;34:395-403.

20. Howard-Jones N. La Organización Panamericana de la Salud: orígenes y evolución. Segunda parte. Crónica de la OMS 1980;34:452-9.

21: Weber M. La ética protestante y el espíritu del capitalismo. Barcelona: Ediciones Península; 1979.

22. Wheatley SC. The politics of philantropy: Abraham Flexner and medical education. Madison: The University of Wisconsin Press; 1988.

23. Bevier G. Discurso del representante de la Fundación Rockefeller, Jefe de la Sección de Uncinariasis, en el homenaje al señor John D. Rockefeller por la Federación de Cafeteros de Colombia". Revista de Higiene (Bogotá) 1932;13(6):189-97.

24. Moll A. Medio siglo de adelanto en medicina y salud pública. Revista de Higiene (Bogotá) 1940;21(1-2): 92-116.

25. Cueto M, editor. Missionaries of science. The Rockefeller Foundation and Latin America. Indianapolis: Indiana University Press; 1994. 
26. Randall SJ. Aliados y distantes. Historia de las relaciones entre Colombia y Estados Unidos desde la Independencia hasta la guerra contra las drogas. Bogotá: Tercer Mundo Editores; 1992.

27. Gast A. Historia de la fiebre amarilla en Colombia. Bogotá: Instituto Nacional de Salud; 1982.

28. Sheps CG. Evolución de las escuelas de salud pública en Estados Unidos a partir de la Segunda Guerra Mundial. In: Bowers JZ, Purcell EF. Escuelas de salud pública: presente y futuro. Buenos Aires: Ateneo; 1976:1-9.

29. Rockefeller Foundation. Annual report. New York: The Rockefeller Foundation; 1916:415-27.

30. García P. La Junta Central de Higiene. Revista de Higiene (Bogotá) 1915;7(93):4-7.

31. Lee V. La danza de los millones. Régimen militar y revolución social en Colombia, 1930-1956. Bogotá: EI Ancora Editores; 1981.

32. Cardona A. Problemática médica antioqueña y su marco sociopolítico en la primera mitad del siglo XX. Medellín: Universidad de Antioquia, AMDA; 1984.

33. Bejarano JA. El capital monopolista y la inversión norteamericana en Colombia. Bogotá: Herrera Hermanos; 1972.

34. Pan-American Sanitary Office. Código Sanitario Panamericano. Bol Ofi Sanit Pan 1925;4(2):45-61.

35. García P. Informe anual de la Junta Central de Higiene al Ministerio de Gobierno. Revista de Higiene, (Bogotá) 1917;8(102):223.

36. García P. Compilación de leyes, decretos, acuerdos y resoluciones vigentes sobre higiene y sanidad en Colombia. Bogotá: Imprenta Nacional; 1932.

37. Miranda $\mathbf{N}$. La medicina colombiana: de la Regeneración a los años de la Segunda Guerra Mundial. En: Tirado A, editor. La nueva historia de Colombia, volumen IV. Bogotá: Planeta; 1989:257-84.

38. García P. Comisión americana para el estudio de la fiebre amarilla. Revista de Higiene (Bogotá) 1917;8 (100):99-104.

39. Franco C. Historia suscinta del saneamiento del suelo emprendida en el país para combatir la anemia tropical o uncinariasis. Revista de Higiene (Bogotá) $1937 ; 18(7): 43-52$.

40. Rockefeller Foundation. Carta dirigida al señor Ministro de Agricultura, firmada por Victor G. Heiser, New York, febrero 19 de 1920. Revista de Higiene (Bogotá) 1937;18(7):15-6.

41. Bevier G. Informe del Director del Departamento de Uncinariasis, sobre los trabajos realizados en el año 1928. En: Colombia, Ministerio de Educación. Memoria del Ministro de Educación, José Vicente Huertas, al
Congreso de 1929. Bogotá: Imprenta Nacional; 1929.

42. Kerr JA. Memorandum sobre las actividades de la Fundación Rockefeller en Colombia, junio de 1935. Revista de Higiene (Bogotá) 1937;18(7):16-21.

43. Ministerio de Trabajo, Higiene y Previsión Social, Colombia. Memoria del Ministro de Trabajo, Higiene y Previsión Social, Arcesio Londoño, al Congreso de 1943. Bogotá: Imprenta Nacional; 1943.

44. Kranaskas A. El Servicio Cooperativo Interamericano de Salud Pública. Mimeografiado. Bogota; 1957.

45. Humphrey GH. Informe de la Misión Médica Unitaria a Colombia. Boletín Clínico (Medellín) 1950;10(10).

46. Lapham M, Gross C, Berson R. Un estudio de la educación médica en Colombia-1953. Antioquia Médica 1954;4:7-8.

47. Ludmerer KM. Learning to heal. The development of American medical education. New York: Basic Books, Inc.; 1985.

48. Pyenson L. Why science may serve political ends: cultural imperialism and the mission to civilize. Berichte zur Wissenschaftsgeschichte 1990;13:6981.

49. Pyenson L. Cultural imperialism and exact sciences revisited. Isis 1993;84:103-8.

50. Berger P, Luckmann T. La construcción social de la realidad. Buenos Aires: Amorrortu Ed.; 1972.

51. Latour B, Woolgar S. Laboratory life. The social construction of scientific facts. London-Beverly Hills: Sage; 1979.

52. Bloor D. Knowledge and social imagery. London: Routledge; 1976.

53. Woolgar S. Interests and explanation in the social study of science. Social Studies of Science 1981;11: 356-94.

54. Woolgar S. Ciencia: abriendo la caja negra. Barcelona: Anthropos; 1991.

55. Ardila M. Cambio de norte? Momentos críticos de la política exterior colombiana. Bogotá: Tercer Mundo Editores; 1991.

56. Mead T, Walker M. Science, medicine and cultural imperialism. New York: St. Martin's Press; 1991.

57. Rahman A. Problems and perspectives. In: Kumar D, editor. Science and empire: essays in Indian context. New Delhi: Anamika Prakashan; 1991.

58. Chambers DW. Locality and science: myths of centre and periphery. En: Lafuente A, Elena A, Ortega ML. Mundialización de la ciencia y cultura nacional. Madrid: Doce Calles/Universidad Autónoma de Madrid; 1993. 